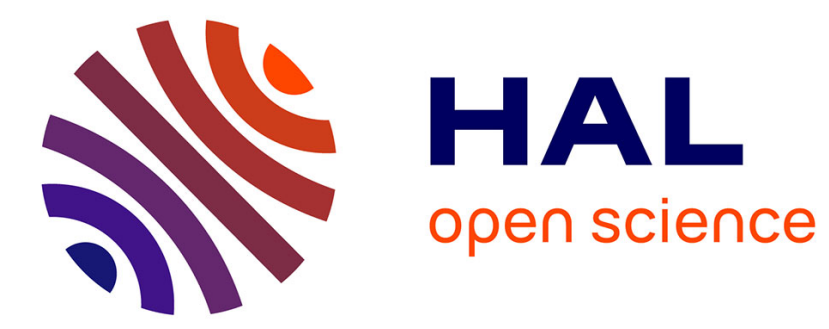

\title{
Large plastic deformation of polycrystals
}

Claude Stolz

\section{To cite this version:}

Claude Stolz. Large plastic deformation of polycrystals. C. Teodosiu. Large Plastic Deformation of Crystalline Aggregates, 376, Springer-Verlag, pp.81-108, 1997, CISM courses and lectures, 10.1007/978-3-7091-2672-1_3. hal-00112492

\section{HAL Id: hal-00112492 \\ https://hal.science/hal-00112492}

Submitted on 31 Oct 2018

HAL is a multi-disciplinary open access archive for the deposit and dissemination of scientific research documents, whether they are published or not. The documents may come from teaching and research institutions in France or abroad, or from public or private research centers.
L'archive ouverte pluridisciplinaire HAL, est destinée au dépôt et à la diffusion de documents scientifiques de niveau recherche, publiés ou non, émanant des établissements d'enseignement et de recherche français ou étrangers, des laboratoires publics ou privés. 


\title{
LARGE PLASTIC DEFORMATION OF POLYCRYSTALS
}

\author{
C. Stolz
}

CNRS-URA 317, Polytechnic, Palaiseau, France

\begin{abstract}
This paper is devoted to the description of the general relationships between micro- and macroscales in non-linear mechanics. After a thermodynamical presentation of these relations, we point out some particular cases of non-linearities, especially the case of polycrystalline aggregates in finite strain. In the case of the single crystal, the energy is well defined in the frame of the crystal lattice, the deformation of which is essentially reversible. The plastic deformation preserves the orientation and the structure of the lattice. In the case of the polycrystal, the constitutive law has the same form as the single-crystal one, the evolution of a triad of vectors is necessary to describe the evolution of the microstructure and to ensure uniqueness of the decomposition of the deformation gradient in a reversible and a plastic part.

The problem of the evolution of the internal state of a single crystal and of a polycrystal is investigated, including the symmetry of the rate boundary-value proilem.
\end{abstract}




\section{GENERAL RELATIONSHIPS BETWEEN MICRO- AND MACRO- MECHANICAL QUANTITIES}

\subsection{Introduction}

We are concerned with a macroscopic description of a small volume element, the local properties of which are known. Inside this element of material, we distinguish between two scales: the microscopic one, where the properties vary from point to point as in a highly heterogeneous body, and the macroscopic one, where the properties are those of a homogeneous continuum.

In order to determine with accuracy the overall behavior of this volume considered as homogeneous, it is essential to define the so-called representative volume element (RVE), which must be small enough to allow us to distinguish the microscopic heterogeneities and sufficiently large to be representative of the overall behavior.

From a thermodynamic viewpoint, it is sufficient to characterize two macroscopic potentials, the first one being the macroscopic free energy and the second one the macroscopic potential of dissipation. With this background, we can describe the state of the body and its evolution.

It is easy to determine the macroscopic quantities from the microscopic ones by an averaging process. It is thus clear that a given microscopic state defines an unique macrostate. Conversely, starting with a macrostate, we must solve a boundary-value problem in order to find the corresponding microscopic state, and the uniqueness of this microstate depends on the concentration process. The determination of the two macroscopic potentials depends obviously on the solution of this boundary-value problem.

For example, it is kwown that the value of the potential energy of a linear elastic system depends only on the value of the displacements and of the tractions given on the boundary. So, in a global description, the potential energy plays the role of the free energy.

The purpose of this paper is mainly to emphazise the role of the global free energy evaluated as the total free energy at an equilibrium state. The determination of the state of equilibrium is a particular boundary-value problem, with particular boundary conditions. In fact, in order to be effective, these conditions must verify some properties, summarized in the concept of concentration process or localization process.

\subsection{The Macrohomogeneity Hypothesis}

We consider a volume $\Omega$, whose material properties vary from point to point like a highly heterogeneous body and we search in a global description for the properties of an equivalent homogeneous continuum. 
To determine with accuracy the overall properties, it is essential to define the representative volume element (RVE), small enough to allow us to distinguish microscopic heterogeneities and sufficiently large to represent the overall behavior. For a polycrystalline aggregate it is necessary to have a minimum of information about the geometry of each constituent and to make assumptions of statistical homogeneity or ergodicity in order to define the RVE in a statistical sense (Kröner, 1980; Willis, 1981; Hashin, 1983).

The problem is to characterize the overall behavior only from a knowledge of the local constitutive equations of each constituent phase of the heterogeneous medium.

With every microscopic quantity $f$, we can associate its macroscopic value $F$ by an averaging process on the RVE, extended eventually to the cases for which the RVE contains holes or rigid inclusions:

$$
F=\frac{1}{\Omega} \int_{\Omega} f d \omega=<f>.
$$

It is clear that in this way a unique macrostate quantity is defined for each microstate.

Conversely, starting with a macrostate, the definition of a suitable corresponding microstate requires complementary information or a concentration process to determine the nearest possible microstate to the real one. For the choice of a representative volume element suitable boundary conditions must be prescribed and must satisfy some requirements (Francfort et al., 1983; Germain et Al., 1983; Stolz, 1987).

For a choice of the boundary conditions, a local stress distribution $\mathbf{t}$ and a local deformation $\varepsilon$ are developed over the volume $\Omega$. Denote by $n$ the unit normal to the boundary $\partial \Omega$ of $\Omega$, and assume that $\partial \Omega=\partial \Omega_{T} \cup \partial \Omega_{u}$, where $\partial \Omega_{T}$ and $\partial \Omega_{u}$ are disjoint parts of $\partial \Omega$, on which the stress vector and respectively the displacement vector are prescribed. The local stresses $t$ verify the equation of equilibrium and the boundary condition:

$$
\operatorname{div} \mathbf{t}=0 \text { over } \Omega, \text { and } \mathbf{t} . n=T^{d} \text { on } \partial \Omega_{T} .
$$

The local deformation derives from a local displacement vector $u$ which verifies in the small strain approximation the relations

$$
\varepsilon_{i j}=\frac{1}{2}\left(\frac{\partial u_{i}}{\partial x_{j}}+\frac{\partial u_{j}}{\partial x_{i}}\right) \text { over } \Omega, \text { and } u=u^{d} \text { on } \partial \Omega_{u} .
$$

We require that the Hill-Mandel macrohomogeneity condition be fulfilled by all fields $\mathbf{t}^{*}$ statically admissible with $\mathbf{T}^{*}=<\mathbf{t}^{*}>$ and by all $\varepsilon^{*}$ kinematically admissible field with $\mathbf{E}^{*}=\left\langle\varepsilon^{*}\right\rangle$, so that

$$
\mathbf{T}^{*}: \mathbf{E}^{*}=<\mathbf{t}^{*}: \boldsymbol{\varepsilon}^{*}>.
$$

In particular, for the study of the macroscopic behavior these boundary conditions can be homogeneous. In this case, one can prescribe homogeneous stress conditions 
$\left(T^{d}=\mathbf{T} \cdot n\right.$ on $\left.\partial \Omega\right)$ or homogeneous strain conditions $(U=\mathbf{E} \cdot y$ for all point $y$ over $\partial \Omega$ ), where $\mathbf{T}$ and $\mathbf{E}$ are two symmetric tensors.

Under these conditions, it is obvious that in the case of homogeneous prescribed stresses, the averaging stress is the applied one, i.e.

$$
\mathbf{T}=<\mathbf{t}>
$$

and the local displacement $u$ fluctuates around the overall displacement $U=\mathbf{E} . y$ such that

$$
\int_{\partial \Omega}(U-u) \otimes n d a=0 .
$$

Then, the relation between microscopic and macroscopic strains can be deduced as

$$
\mathbf{E}=<\varepsilon>\text {. }
$$

Dually, if an uniform strain is prescribed on the boundary $(u=U=\mathbf{E} \cdot y$ over $\partial \Omega)$, it is clear that

$$
\mathbf{E}=\langle\varepsilon\rangle
$$

and the microstresses are such that

$$
\int_{\partial \Omega}(<\mathbf{t}>-\mathbf{t}) \cdot n \otimes y d a=0, \text { hence } \mathbf{T}=<\mathbf{t}>.
$$

Some other boundary conditions can be used. In particular, for periodic structures (Sanchez Palencia 1974, Suquet 1982) the elementary cell generates the geometry of the whole by periodicity, all the local fields are periodic functions and the Hill-Mandel macrohomogeneity condition is a necessary condition resulting from periodicity.

The construction of the macroscopic constitutive law from the microscopic behavior can proceed as follows:

- starting from some macroscopic quantities, then

- using a concentration or localization process to determine local fields, and finally

- determining the missing macroscopic quantities by the averaging process.

\subsection{Mode and Process of Localization}

For a given choice of a representative volume element suitable boundary conditions must be prescribed to solve the problem of localization. These conditions must satisfy some requirements in order to be effective:

- the definition of an averaging process (average over a volume or statistical averaging); the average value of the field $f$ will be denoted by $F=\langle f\rangle$; 
- the equations of continuum mechanics (equilibrium, compatibility of the strain and boundary conditions) must be verified;

- the boundary conditions must verify the hypothesis of macrohomogeneity in the sense of Hill-Mandel.

For example, we can prescribe a mode in homogeneous stresses $\left(T^{d}=\mathbf{T} . n\right.$ on $\left.\partial \Omega\right)$, or in homogeneous strains $(U=\mathbf{E} . y$ for $y \in \partial \Omega)$, or a mode associated with periodicity conditions.

Generally, for a given choice of boundary conditions local stresses $\mathbf{t}$ and strains $\varepsilon$ are developed over $\Omega$. These two fields must satisfy the mechanical equations:

- the micro stress field $\mathbf{t}$ satisfies the equilibrium equations, the traction boundary conditions and the average property

$$
\mathbf{T}=<\mathbf{t}>,
$$

- the micro strain field satisfies the compatibility equation, the diaplacement boundary conditions of the mode of localization and the average property

$$
\mathbf{E}=\langle\varepsilon>\text {. }
$$

The stress field $\mathbf{t}$ and the strain field $\varepsilon$ satisfying these conditions will be said to be respectively statically admissible (S.A.) with $\mathbf{T}$ and kinematically admissible (K.A.) with $\mathbf{E}$ in the mode of localization.

- The boundary conditions must fulfil the Hill-Mandel macrohomogeneity condition:

For any stress field $\mathbf{t}$ which is $S . A$. with $\mathbf{T}$ in the mode and any field $\boldsymbol{\varepsilon}$ which is $K . A$. with $\mathbf{E}$ in the mode, the intermal power is conserved i.e.

$$
\mathbf{T}: \mathbf{E}=\langle\mathbf{t}: \boldsymbol{\varepsilon}>
$$

- The process of localization defined by a mode of localization and a constitutive law must ensure the existence and uniqueness of the microscopic fields.

These properties ensure the possibility of the determination of the general relationships between microscale and macroscale. In particular, we can deduce the form of the macroscopic constitutive law in the following way: for the prescribed macroscopic quantities we solve the boundary-value problem associated with the process of localization, we determine the local fields and finally, using the averaging process, we find the unknown macroscopic quantities. 


\subsection{The Case of Systems in Equilibrium}

The local behavior is given by the local free energy density $\psi(\varepsilon, \alpha, T)$, where $\varepsilon$ is the strain tensor, $\alpha$ represents a set of internal variables and $T$ is the absolute temperature. 'The state equations are given by

$$
\mathbf{t}=\frac{\partial \psi}{\partial \boldsymbol{\varepsilon}}, s=-\frac{\partial \psi}{\partial T}, A=-\frac{\partial \psi}{\partial \alpha} .
$$

$A$ is the thermodynamical force associated with the evolution of $\alpha$ and $s$ is the entropy. When we have no viscosity, $t$ is the Cauchy stress and is related to the equilibrium state via the solution of a boundary-value problem. In the following sections we describe only isothermal processes, so we do not consider the thermal problem.

The equations of the boundary-value problem. If we prescribe a macroscopic strain $\mathbf{E}=\langle\varepsilon\rangle$, for a given distribution of internal parameters $\alpha$ we must find the local fields $\mathbf{t}$ as functions of $\mathbf{E}$ and $\alpha$ by solving the following problem.

Find a displacement field $u$ satisfying the equation

$$
\mathbf{E}=\left\langle\boldsymbol{\varepsilon}>, \mathbf{t}=\frac{\partial \psi}{\partial \boldsymbol{\varepsilon}}, \operatorname{div} \mathbf{t}=0,\right.
$$

and the boundary conditions of the mode.

Denoting then by $\Psi$ the macroscopic free energy density $\Psi(\mathbf{E}, \alpha)=\langle\psi(\varepsilon(u), \alpha)>$, where $\varepsilon(u)$ is the strain associated with the solution of the above boundary-value problem. We deduce the equations of state by using the Hill-Mandel macrohomogeneity condition

$$
\frac{\partial \Psi}{\partial \mathbf{E}}=<\frac{\partial \psi}{\partial \boldsymbol{\varepsilon}}: \frac{\partial \boldsymbol{\varepsilon}}{\partial \mathbf{E}}>=<\mathbf{t}:\left(\mathbf{I}+\frac{\partial \boldsymbol{\eta}}{\partial \mathbf{E}}\right)>.
$$

Noting that $\varepsilon$ is written as $\mathbf{E}+\boldsymbol{\eta}$, where $\boldsymbol{\eta}$ is a strain kinematically admissible with zero in the mode, we have $\partial \boldsymbol{\eta} / \partial \mathbf{E}=\mathbf{0}$, and hence the macroscopic stress $\mathbf{T}$ is related to the macroscopic strain by the state equation

$$
<\mathbf{t}>=\mathbf{T}=\frac{\partial \Psi}{\partial \mathbf{E}} .
$$

The macrostress at equilibrium is defined in the same way as the microstress, owing to the definition of the macroscopic thermodynamic potential $\Psi$.

The other relations are expressed by

$$
A \bullet \delta \alpha=-\int_{\Omega} \frac{\partial \psi}{\partial \alpha} \delta \alpha d \omega=-\frac{\partial \Psi}{\partial \alpha} \bullet \delta \alpha,
$$

then the internal state in a global description for the system is defined by the value of $\alpha(x)$ in each point of $\Omega$, so the internal state is defined by a field of internal variables. 
Case of a macrohomogeneous body. For linear elasticity, it is well known that the macroscopic elastic modulus has not the same value when macrohomogeneous strain or stress conditions are prescribed on the boundary $\partial \Omega$. But when the body can be considered as macrohomogeneous in the sense of Hill $(1966,1967)$ and Mandel (1964) the difference between the two estimates vanishes. The volume of the VER is sufficiently large to contain enough heterogeneities to consider the whole specimen as a homogeneous body at the macroscale.

Assuming that all constituent phases are linear elastic, the local free energy is

$$
\psi=\frac{1}{2} \varepsilon: c: \varepsilon,
$$

where $c$ depends on the point $x$ de $\Omega$. The solution of the boundary-value problem of the equilibrium is the minimum of the potential energy of the system, when one prescribes the boundary condition as a homogeneous displacement over $\partial \Omega$, the potential energy $\Psi$ depends only of the given $\mathbf{E}$, and of the spatial distribution of the mechanical phases.

When homogeneous boundary conditions are prescribed in stress $\left(T^{d}=\mathbf{T} . n\right.$ on $\partial \Omega$, with $\mathbf{T}$ a symmetric tensor) the local stress $\mathbf{t}$ is obtained as the solution of an elastic linear problem, and this proves the existence of a concentration tensor $\mathcal{A}_{i j p q}$ such that

$$
t_{i j}=\mathcal{A}_{i j p q} T_{p q}
$$

Actually, this tensor $\mathcal{A}$ is the elastic concentration tensor introduced by Mandel (1964) in the case of a localization process in homogeneous macrostresses.

As $<\mathbf{t}>=\mathbf{T}$ for all prescribed $\mathbf{T}$, we have

$$
<\mathcal{A}_{i j p q}>=\frac{1}{2}\left(\delta_{i p} \delta_{j q}+\delta_{i q} \delta_{j p}\right) .
$$

For fixed subscripts $(p, q), \mathcal{A}_{i j p q}$ satisfies the equilibrium equations

$$
\mathcal{A}_{i j p q, j}=0 \text {, on } \Omega \text {. }
$$

and homogeneous boundary conditions

$$
\mathcal{A}_{i j p q} n_{j}=\frac{1}{2}\left(\delta_{i p} \delta_{j q}+\delta_{i q} \delta_{j p}\right) n_{j} \text { over } \partial \Omega,
$$

whereas the deformation $\varepsilon_{i j}=s_{i j k l} \mathcal{A}_{k l p q}$ satisfies the condition of compatibility.

Moreover, from the local behavior, we can define the following relations

$$
\begin{gathered}
\mathbf{t}=c: \boldsymbol{\varepsilon}=c: \mathcal{B}: \mathbf{E}, \\
\mathbf{t}=\mathcal{A}: \mathbf{T}=\mathcal{A}: \mathcal{C}: \mathbf{E}, \\
c: \mathcal{B}=\mathcal{A}: \mathcal{C}, \mathcal{B}: \mathcal{S}=s: \mathcal{A}, \\
\mathcal{C}=\left\langle\mathcal{B}^{t}: c: \mathcal{B}>, \mathcal{S}=\left\langle\mathcal{A}^{t}: s: \mathcal{A}>=\mathcal{C}^{-1} .\right.\right.
\end{gathered}
$$


Elastic constitutive laws. For more general constitutive behavior, we can define the instantaneous elastic moduli by considering small perturbations of the state of equilibrium at fixed internal parameters.

The solution associated with a variation of the macroscopic strain $\mathbf{E}$ is then the elastic response. The problem to be solved is the problem of the evolution of an heterogeneous medium. Denoting the local moduli by $c$, we have

$$
c=\frac{\partial^{2} \psi}{\partial \varepsilon \partial \varepsilon}
$$

and the evolution is given by solving the rate boundary-value problem

$$
\dot{\mathbf{E}}=<\dot{\boldsymbol{\varepsilon}}>, \operatorname{div} \dot{\mathbf{t}}=0, \quad \dot{\mathbf{t}}=c: \dot{\boldsymbol{\varepsilon}} .
$$

This problem is a problem of linear elasticity, which can be described by the introduction of a stress or strain concentration tensor, $\mathcal{A}$ or $\mathcal{B}$, as influence tensors. The solution of the problem of heterogeneous elasticity is written as

$$
\dot{\varepsilon}=\mathcal{B}: \dot{\mathbf{E}}, \dot{\mathbf{t}}=\mathcal{A}: \dot{\mathbf{T}} .
$$

These tensors are associated with the local elastic modulus and the macroscopic modulus satisfies

$$
S=\left\langle\mathcal{A}^{t}: s: \mathcal{A}>.\right.
$$

Note that for fixed (p q), $\mathcal{A}_{i j p q}$ is a statically admissible field in the mode with the identity and $\mathcal{B}_{i j p q}$ is a kinematically admissible field with the identity. These properties can be resumed by

$$
<\mathcal{A}\rangle=\mathcal{I},\langle\mathcal{B}\rangle=\mathcal{I} .
$$

The identity tensor is here $\mathcal{I}_{i j p q}=\frac{1}{2}\left(\delta_{i p} \delta_{j q}+\delta_{j q} \delta_{i p}\right)$.

\subsection{Decomposition of the Macroscopic Strain in Elasto-Plasticity}

The decomposition of the strain is determined with respect to the reversibility. Let $\mathbf{T}$ be the real macrostress and $t$ the corresponding microscopic stress. The local solution for a purely elastic behavior is $\mathbf{t}_{E}=\mathcal{A}: \mathbf{T}$. The stress field $\mathbf{r}=\mathbf{t}-\mathbf{t}_{E}$ is then self-equilibrated.

In small strains, the total deformation $\varepsilon$ is the sum of the elastic strain $\varepsilon_{e}$, related to $\mathbf{t}$ by the elastic constitutive law $\varepsilon_{e}=s: \mathbf{t}$, and of the anelastic strain $\varepsilon_{p}$. Then, the stress field $\mathbf{r}$ is related to the local strain $\varepsilon_{i r}$ by

$$
\varepsilon_{i r}=s: \mathbf{r}+\varepsilon_{p},
$$

which satisfies the compatibility conditions. 
By definition the macroscopic elastic strain $\mathbf{E}_{E}$ is the strain obtained by purely elastic unloading. The local strains $\varepsilon$ and $\varepsilon_{E}=s: \mathbf{t}_{E}$ are two kinematically admissible fields respectively with $\mathbf{E}$ and $\mathbf{E}_{E}$ in the mode of localization. These strains verify the Hill-Mandel condition.

For any stresses $\mathbf{t}$ statically admissible with $\langle\mathbf{t}\rangle$ in the mode of localization with the particular choice $\mathbf{t}=\mathcal{A}:\langle\mathbf{t}\rangle$, we obtain

$$
\begin{aligned}
\mathbf{E}_{E} & =\left\langle\mathcal{A}^{t}: \boldsymbol{\varepsilon}_{E}\right\rangle, \\
\mathbf{E} & =\left\langle\mathcal{A}^{t}: \boldsymbol{\varepsilon}\right\rangle
\end{aligned}
$$

Then, for the elastic response $\varepsilon_{E}=s: \mathbf{t}_{E}$, where $\mathbf{t}_{E}=\mathcal{A}:\left\langle\mathbf{t}_{E}>=\mathcal{A}: \mathbf{T}\right.$, the definition of the macroscopic modulus can be deduced as

$$
\mathcal{S}=\left\langle\mathcal{A}^{t}: s: \mathcal{A}>.\right.
$$

The difference $\mathbf{E}-\mathbf{E}_{E}$ is a kinematically admissible field associated with the anelastic part of the macroscopic strain $\mathbf{E}_{p}=\mathbf{E}-\mathbf{E}_{E}$, and hence

$$
\mathbf{E}_{p}=<\mathcal{A}^{t}: \varepsilon_{i r}>\text {. }
$$

Since $\mathbf{r}$ is a self-equilibrated stress field and $s: \mathcal{A}$ is a kinematically admissible field, the classical results of the definition of the plastic macroscopic part is then recovered:

$$
\mathbf{E}_{p}=<\mathcal{A}^{t}: \varepsilon_{p}>\text {. }
$$

It is obvious that all these equations hold also for the time derivatives of the quantities involved. In particular, we have

$$
\dot{\mathbf{E}}_{p}=<\mathcal{A}^{t}: \dot{\varepsilon}_{p}>.
$$

This definition derives from the kinematical aspect of the evolution, its thermodynamic signification must be investigated.

Global domain of elasticity and dissipation rate. At the local level we assume that at each point $x$ of the RVE an elastic domain $C(x)$ is given by means of a convex function $f$ :

$$
C(x)=\{\mathbf{t} \mid f(\mathbf{t})<0\} .
$$

At the macroscopic level the elastic domain $C_{\mathbf{T}}$ is determined as follows.

Let $\mathbf{T}$ and $\mathbf{T}^{*}$ be two macroscopic stresses belonging to $C_{\mathbf{T}}$. The path $\mathbf{T}-\mathbf{T}^{*}$ is an elastic path, which implies that the corresponding local path $\mathbf{t}-\mathbf{t}^{*}$ is an elastic path, too.

As $\mathbf{t}(x) \in C(x)$ for all points $x$ of the RVE, $\mathbf{t}^{*}$ verifies the same property. Thus, we have

$$
\mathbf{t}-\mathbf{t}^{*}=\mathcal{A}:\left(\mathbf{T}-\mathbf{T}^{*}\right)
$$

This property induces a normality rule at the macroscopic level if such a rule holds at the local scale. 
Normality rule. The normality rule is expressed in the form of the principle of maximal plastic work of Hill: At each point of the RVE, if $\mathbf{t}(x) \in C(x)$, we have for any $\mathbf{t}^{*}(x) \in C(x)$,

$$
\left(\mathbf{t}-\mathbf{t}^{*}\right): \dot{\varepsilon}_{p} \geq 0 .
$$

Since $\mathbf{T} \in C_{\mathbf{T}}$, we associate to any $\mathbf{T}^{*} \in C_{\mathbf{T}}$ the local field $\mathbf{t}^{*}$ defined by

$$
\mathbf{t}-\mathbf{t}^{*}=\mathcal{A}:\left(\mathbf{T}-\mathbf{T}^{*}\right) .
$$

Such a field $\mathbf{t}^{*}$ is an element of $C(x)$ for all $\mathrm{x}$. Therefore taking the average of the inequality we obtain

$$
\left(\mathbf{T}-\mathbf{T}^{*}\right):<\mathcal{A}^{t}: \dot{\varepsilon}_{p}>\geq 0,
$$

which is equivalent to

$$
\left(\mathbf{T}-\mathbf{T}^{*}\right): \dot{\mathbf{E}}_{p} \geq 0 .
$$

The macroscopic domain of elasticity is then convex, since it is the intersection of the convex domains $C(x)$.

Dissipation rate. For elastoplasticity, another characterization is obtained when the dissipation rate is studied. Firstly, one considers at the local level an elastic-perfectly plastic material. The density of energy being $\psi\left(\varepsilon, \varepsilon_{p}\right)=\psi\left(\varepsilon-\varepsilon_{p}\right)$, the dissipation rate takes the form

$$
D_{p}=<\mathbf{t}: \dot{\varepsilon}_{p}>\geq 0 .
$$

Expressing it in terms of macroscopic quantities, we find

$$
D_{p}=\mathbf{T}: \dot{\mathbf{E}}_{p}+<\mathbf{r}: \dot{\varepsilon}_{p}>\geq 0 .
$$

The macroscopic free energy takes the form given in terms of stress

$$
\Psi=<\psi>=\frac{1}{2} \mathbf{T}: S: \mathbf{T}+\frac{1}{2}<\mathbf{r}: s: \mathbf{r}>,
$$

and the dissipation rate shows the role played by the energy embedded in the residual stresses

$$
D_{p}=\mathbf{T}: \dot{\mathbf{E}}_{p}-\frac{1}{2}<\frac{d}{d t} \mathbf{r}: s: \mathbf{r}>\geq 0 .
$$

This result is due to the fact that $\dot{\boldsymbol{\varepsilon}}_{\text {ir }}$ is a kinematically admissible field, and that the residual stresses $\mathbf{r}$ are self-equilibrated

$$
0=\left\langle\mathbf{r}: \dot{\varepsilon}_{i r}>=<\mathbf{r}: s: \dot{\mathbf{r}}+\mathbf{r}: \dot{\varepsilon}_{p}>.\right.
$$


Macroscopic viscoplastic potential. This equation and the definition of the macroscopic convex of elasticity show that a macroscopic viscoplastic potential $\Omega$ exists if a local one $\omega$ is given. $\Omega$ is defined by the averaging process as

$$
\Omega(\mathbf{T}, \mathbf{r})=<\omega(\mathbf{t})>\text {. }
$$

The local normality rule is expressed by

$$
\dot{\varepsilon}_{p}=\frac{\partial \omega}{\partial \mathrm{t}} \text {. }
$$

Since $\mathbf{t}=\mathcal{A}: \mathbf{T}+\mathbf{r}$, it is clear that the normality rule results from the set of equations

$$
\begin{aligned}
& \frac{\partial \Omega}{\partial \mathbf{r}}=\frac{\partial \omega}{\partial \mathbf{r}}=\dot{\boldsymbol{\varepsilon}}_{p} \\
& \frac{\partial \Omega}{\partial \mathbf{T}}=\left\langle\mathcal{A}^{T}: \frac{\partial \omega}{\partial \mathbf{r}}\right\rangle=\dot{\mathbf{E}}_{p} .
\end{aligned}
$$

Generalization to other behaviors. These equations can be extended to more complicated behaviors, by using the definition of standard generalized materials.

For example, to describe hardening, the free energy density is supposed to possess the following expression

$$
\psi\left(\varepsilon, \varepsilon_{p}, \alpha\right)=\frac{1}{2}\left(\varepsilon-\varepsilon_{p}\right): c:\left(\varepsilon-\varepsilon_{p}\right)+w_{b}\left(\varepsilon_{p}, \alpha\right),
$$

with the associated equations of state

$$
\mathbf{t}=\frac{\partial \psi}{\partial \boldsymbol{\varepsilon}}, \boldsymbol{\sigma}=-\frac{\partial \psi}{\partial \boldsymbol{\varepsilon}_{p}}=\mathbf{t}-\frac{\partial w_{b}}{\partial \boldsymbol{\varepsilon}_{p}}, A=-\frac{\partial \psi}{\partial \alpha}
$$

If there is no viscosity effects, $t$ is the stress at equilibrium. If the domain of elasticity is defined by

$$
f(\boldsymbol{\sigma}, A)=\sqrt{\frac{1}{2} \mathbf{s}: \mathbf{s}}+A-k_{o} \leq 0,
$$

where $\mathbf{s}$ is the deviatoric part of $\sigma$,we recognize immediatly three particular cases :

- when $\mathrm{w}_{b}=0$, i.e.in the case of perfect plasticity, the normality rule gives the rate of plastic strain

$$
\dot{\varepsilon}_{p}=\mu \frac{\partial f}{\partial \mathbf{s}}, \mu f=0 \text { and } f \leq 0, \mu \geq 0,
$$

- when $\mathrm{w}_{b}=\frac{1}{2} \varepsilon_{p}: H: \varepsilon_{p}$, i.e. in the case of linear hardening, the normality rule defines the rate of internal variables as :

$$
\dot{\varepsilon}_{p}=\mu \frac{\partial f}{\partial \mathbf{s}}, \mu f=0 \text { and } f \leq 0, \mu \geq 0,
$$


- when $\mathrm{w}_{b}=H(\alpha)$, i.e. in the case of isotropic hardening, the evolution of the hardening $\mu$ is defined by the normality rule

$$
\begin{array}{r}
\dot{\boldsymbol{\varepsilon}}_{p}=\mu \frac{\partial f}{\partial \mathbf{s}}=\frac{1}{2} \frac{\mu \mathbf{s}}{\sqrt{\frac{1}{2} \mathbf{s}: \mathbf{s}}}, \dot{\alpha}=\mu \frac{\partial f}{\partial A}=\sqrt{2 \dot{\varepsilon}_{p}: \dot{\varepsilon}_{p}} \\
\mu f=0 \text { and } f \leq 0, \mu \geq 0 .
\end{array}
$$

In the general case, we can combine isotropic and kinematic hardening. The dissipation rate is

$$
d_{p}=\boldsymbol{\sigma}: \dot{\varepsilon}_{p}+A \dot{\alpha} \geq 0 .
$$

By the averaging process, with the decomposition of $\sigma=\mathcal{A}: \mathbf{T}+\sigma_{p}$, we can show that

$$
D_{p}=\left\langle d_{p}>=\mathbf{T}:\left\langle\mathcal{A}^{t}: \dot{\varepsilon}_{p}>+<\sigma_{p}: \dot{\varepsilon}_{p}>+<A \dot{\alpha}>\geq 0,\right.\right.
$$

where $\sigma_{p}=r-\frac{\partial w_{h}}{\partial \varepsilon_{p}}$.

We can show then that the global viscoplastic potential is :

$$
\Omega\left(\mathbf{T}, \boldsymbol{\sigma}_{p}, A\right)=\int_{\Omega} \omega(\boldsymbol{\sigma}, A) d \omega
$$

where $\sigma=\mathcal{A}: T+\sigma_{p}$. The normality rule at the local state

$$
\dot{\varepsilon}_{p}=\frac{\partial \omega}{\partial \sigma}, \dot{\alpha}=\frac{\partial \omega}{\partial A}
$$

implies that

$$
\begin{aligned}
\frac{\partial \Omega}{\partial \boldsymbol{\sigma}_{p}} & =\frac{\partial \omega}{\partial \boldsymbol{\sigma}_{p}}=\dot{\varepsilon}_{p}, \\
\frac{\partial \Omega}{\partial A} & =\frac{\partial \omega}{\partial A}=\dot{\alpha} \\
\frac{\partial \Omega}{\partial \mathbf{T}} & =\left\langle\mathcal{A}^{t}: \frac{\partial \omega}{\partial \boldsymbol{\sigma}_{p}}>=\dot{\mathbf{E}}_{p} .\right.
\end{aligned}
$$

These equations are true for plasticity, as a limit case of viscoplasticity. In fact, because the potential is convex, we can use the definition of the dissipative function $d\left(\dot{\varepsilon}_{p}, \dot{\alpha}\right)$ instead of the potential of dissipation, such that

$$
d\left(\dot{\varepsilon}_{p}, \dot{\alpha}\right)=\underset{\sigma, A}{\operatorname{Sup}}\left(\omega(\boldsymbol{\sigma}, A)-\boldsymbol{\sigma}: \dot{\varepsilon}_{p}-A \dot{\alpha}\right)
$$

and then

$$
\boldsymbol{\sigma}=\frac{\partial d}{\partial \dot{\varepsilon}_{p}}, A=\frac{\partial d}{\partial \alpha} .
$$


The characterization of this normality law, which is always true is

$$
\begin{gathered}
(\boldsymbol{\sigma}, A) \in \partial d\left(\dot{\varepsilon}_{p}, \dot{\alpha}\right) \text { if and only if } \forall\left(\varepsilon^{*}, \alpha^{*}\right) \text { admissible } \\
d\left(\dot{\varepsilon}_{p}, \dot{\alpha}\right)+\boldsymbol{\sigma}:\left(\varepsilon^{*}-\dot{\varepsilon}_{p}\right)+A\left(\alpha^{*}-\dot{\alpha}\right) \leq d\left(\varepsilon^{*}, \alpha^{*}\right)
\end{gathered}
$$

This definition is generalized on the macroscopic level by means of the definition of the dissipative function with the fields $\dot{\varepsilon}_{p}, \dot{\alpha}$ as argument

$$
\begin{gathered}
D\left(\dot{\varepsilon}_{p}, \dot{\alpha}\right)=<d\left(\dot{\varepsilon}_{p}, \dot{\alpha}\right)> \\
D\left(\dot{\varepsilon}_{p}, \dot{\alpha}\right)+<\boldsymbol{\sigma}:\left(\varepsilon^{*}-\dot{\varepsilon}_{p}\right)>+<A\left(\alpha^{*}-\dot{\alpha}\right)>\leq D\left(\varepsilon^{*}, \alpha^{*}\right),
\end{gathered}
$$

with the determination

$$
\begin{aligned}
(\boldsymbol{\sigma}, A) & \in \partial D\left(\dot{\varepsilon}_{p}, \dot{\alpha}\right) \text { if and only if } \forall\left(\varepsilon^{*}, \alpha^{*}\right) \text { admissible, } \\
D\left(\dot{\varepsilon}_{p}, \dot{\alpha}\right)+ & <\boldsymbol{\sigma}:\left(\varepsilon^{*}-\dot{\varepsilon}_{p}\right)>+<A\left(\alpha^{*}-\dot{\alpha}\right)>\leq D\left(\varepsilon^{*}, \alpha^{*}\right) .
\end{aligned}
$$

Taking into account that $\mathbf{r}$ is self-equilibrated, the dissipation function is reduced to $D\left(\dot{\mathbf{E}}_{p}, \dot{\varepsilon}_{p}-\dot{\mathbf{E}}_{p}, \dot{\alpha}\right)$, where we have separated the overall definition of an irreversible strain, and the part due to the change of the internal state which is the source of two kinds of hardening

- the hardening due to the incompatibility of the plastic strain,

- the self-hardening of each constituent.

The hardening is described in the energy embedded in the residual stresses and in the self-hardening energy

$$
\Psi=<\psi>=\frac{1}{2} \mathbf{T}:\left\langle\mathcal{A}^{t}: s: \mathcal{A}>: \mathbf{T}+\frac{1}{2}<\mathbf{r}: s: \mathbf{r}>+\Psi_{b} .\right.
$$

The total dissipation has the expression

$$
D=\mathbf{T}: \dot{\mathbf{E}}_{p}-\frac{d}{d t}\left(\frac{1}{2}<\mathbf{r}: s: \mathbf{r}>+\Psi_{b}\right) \geq 0,
$$

which emphasizes the role of the embedded energy in the hardening.

\subsection{Generalization to Finite Strain}

Consider a body $\Omega$ in its initial state. The body moves under a given loading. The material point initially in $X$ has actual position $x$, and the deformation gradient of the motion $x=x(X, t)$ is then 


$$
\mathbf{f}=\frac{\partial x}{\partial X}
$$

which verifies obviously the average equality

$$
<\mathbf{f}>=\frac{1}{\Omega} \int_{\Omega_{o}} \frac{\partial x}{\partial X} d \omega=\frac{1}{\Omega} \int_{\partial \Omega_{o}} x \otimes N d S=\mathbf{F} .
$$

If the free energy density is a given function of the actual deformation for each microelement from its natural state, we must take into account that at the stress free state of the body $\Omega$ each microelement has constraints $\mathbf{f}_{o}$ such that the microelement is transformed as $\overline{\mathbf{f}}=\mathbf{f}$. $\mathbf{f}_{o}$. The free energy $\psi$ density is then a function of $\overline{\mathbf{f}}$, and of the internal parameters $\alpha$.

The total $\overline{\mathbf{f}}$ is not kinematically admissible, because of the presence of the internal incompatibility of the irreversible strains (it is not the derivative of a displacement field).

As pointed out before, the global gradient $\mathbf{F}$ is related to the microscopic $\mathbf{f}$, such that $\overline{\mathbf{f}}=\mathbf{f} . \mathbf{f}_{o}$, where $\mathbf{f}_{o}$ is the deformation of the microelement from its natural state to the initial state of the body. Inside the body, in its initial state, a self-equilibrated stress field $\boldsymbol{\pi}^{\circ}$ is related to the state of local deformation $\mathbf{f}_{o}$.

As in the case of small strains, we must define now a mode of localization. Consequently some requirements are the same, but the macrohomogeneity lemma is modified. The mode of localization is defined by a boundary-value problem, such that all mechanical equations are verified.

The state of stresses are in equilibrium with respect to the boundary conditions in traction, the strain field is kinematical admissible with the displacement boundary conditions. The boundary conditions are compatible with the average process and must be such that the Hill-Mandel principle of macrohomogeneity be fulfilled.

Macrohomogeneity in finite deformation. For all displacement $u$ which is K.A. with the boundary conditions and all nominal stress field $\theta$ in equilibrium with the boundary conditions defined by the mode of localization we have

$$
<\boldsymbol{\theta}^{i \alpha} \mathbf{f}_{\alpha}^{j}>=<\boldsymbol{\theta}^{i \alpha}><\mathbf{f}_{\alpha}^{j}>.
$$

Global free energy. The global free energy is then $\bar{\rho} \Psi=\langle\rho \psi\rangle$, and we can prove the thermodynamical relation

$$
<\boldsymbol{\theta}>=\bar{\rho} \partial \psi / \partial \mathbf{F}=\boldsymbol{\Theta} .
$$

For a given state $\mathbf{F}$, we must define a displacement $u$ kinematically admissible with the given $\mathbf{F}$, such that $\mathbf{f}=\mathbf{I}+G r a d u$, the nominal stresses $\boldsymbol{\theta}=\rho \partial \psi / \partial \mathbf{f}$ being in 
equilibrium. At this equilibrium state, the global free energy is a function of $\mathbf{F}$, and of the internal state $\alpha$.

$$
\bar{\rho} \Psi\left(\mathbf{F}, \mathbf{f}_{o}, \alpha\right)=<\rho \psi\left(\mathbf{f} . \mathbf{f}_{o}, \alpha\right)>,
$$

and

$$
\bar{\rho} \frac{\partial \Psi}{\partial \mathbf{F}}=<\boldsymbol{\rho} \frac{\partial \psi}{\partial \mathbf{f}}: \frac{\partial \mathbf{f}}{\partial \mathbf{F}}>=<\boldsymbol{\theta}><\frac{\partial \mathbf{f}}{\partial \mathbf{F}}>,
$$

but

$$
\mathbf{f}=\mathbf{F}+\text { Grad } \tilde{u},
$$

where $\tilde{u}$ is kinematically admissible with 0 , because $\langle\mathbf{f}>=\mathbf{F}$, and in a similar way as in the small perturbation approximation, the global potential $\Psi$ gives the stresses at the equilibrium.

If we take into account the principle of objectivity, the free energy density is of the form $\psi=\tilde{w}(\boldsymbol{\Delta}(\overline{\mathbf{f}}), \alpha, T)$, the state of deformation is defined by the Cauchy-Green tensor or the Green-Lagrange tensor,

$$
\Delta(\mathbf{f})=\frac{1}{2}\left(\mathbf{f}^{T} . \mathbf{f}-\mathbf{I}\right)=\mathbf{f}_{o}^{T} \cdot \Delta(\overline{\mathbf{f}}) \cdot \mathbf{f}_{o}+\Delta\left(\mathbf{f}_{o}\right),
$$

and the state equations are related to the actual state of stresses defined in the actual state by $\mathbf{t}$

$$
\frac{\pi}{\rho_{o}}=\frac{\partial \tilde{w}}{\partial \Delta(\mathbf{f})}=\mathbf{f}_{o} \cdot \frac{\overline{\boldsymbol{\pi}}}{\tilde{\rho}} \cdot \mathbf{f}_{o}^{T}=\mathbf{f}^{-1} \cdot \frac{\mathbf{t}}{\rho} \cdot \mathbf{f}^{-T} .
$$

The nominal stresses are then

$$
\boldsymbol{\theta}=\frac{\partial \psi}{\partial \mathbf{f}}=\boldsymbol{\pi} \cdot \mathbf{f}^{T}=\mathbf{f}^{-1} \cdot \mathbf{t} \operatorname{det} \mathbf{f} .
$$

If we have uniqueness of the local solution, the local deformation is a function of the macroscopic state $\mathbf{F}$

$$
\mathbf{f}=B(\mathbf{F}, X) .
$$

When some evolution occurs, we define the pseudo modulus $\mathcal{C}$ as

$$
\dot{\Theta}=\bar{\rho} \frac{\partial^{2} W}{\partial \mathbf{F} \partial \mathbf{F}}: \dot{\mathbf{F}}=\mathcal{C}: \dot{\mathbf{F}},
$$

and the local moduli by

$$
c^{\circ}=\rho \frac{\partial^{2} \psi}{\partial \mathbf{f} \partial \mathbf{f}} .
$$

We have then the relations

$$
\mathcal{C}=\bar{\rho} \frac{\partial^{2} W}{\partial \mathbf{F} \partial \mathbf{F}}=<\rho \frac{\partial B}{\partial \mathbf{F}}^{T}: c^{o}: \frac{\partial B}{\partial \mathbf{F}}>,
$$

and the following general properties. 
For all stresses $\mathbf{t}$ which are S.A. with $\mathbf{T}$, and all velocities $v$ which are K.A. with $V=G r a d V \cdot X$,

$$
\begin{aligned}
\operatorname{GradV} & =\langle\operatorname{grad} v> \\
\mathbf{T} & =\langle\mathbf{t}>, \\
\mathbf{T}: \operatorname{Grad} V & =\langle\mathbf{t}: \operatorname{grad} v>. \\
\bar{\rho} D_{c} \frac{\mathbf{T}}{\bar{\rho}} & \left.=<\rho D_{c} \frac{\mathbf{t}}{\rho}\right\rangle, \\
\dot{\boldsymbol{\Theta}} & =<\dot{\boldsymbol{\theta}}>,
\end{aligned}
$$

where the convective rate is defined as

$$
D_{c} \mathbf{a}=\dot{\mathbf{a}}-\operatorname{grad} v \mathbf{a}-\mathbf{a} \cdot \operatorname{grad}^{t} v .
$$

Tangent moduli and localization. The rate of the nominal stress verifies the momentum equations and the tangent constitutive law in the following form

$$
\dot{\boldsymbol{\theta}}=\rho D_{c} \frac{\mathbf{t}}{\rho}+\mathbf{t} \cdot g r a d^{t} v,
$$

where $D_{c} \frac{\mathbf{t}}{\rho}=\Lambda^{o}: \operatorname{grad} v$, then $\dot{\boldsymbol{\theta}}=c: \operatorname{grad} v$. For the overall behavior, we obtain

$$
\dot{\boldsymbol{\Theta}}=\bar{\rho} D_{c} \frac{\mathbf{T}}{\bar{\rho}}+\mathbf{T} \cdot G r a d^{T} V,
$$

with $D_{c} \frac{\mathbf{T}}{\bar{\rho}}=\Lambda: \mathbf{D}$, then $\dot{\boldsymbol{\Theta}}=\mathcal{C}: G r a d V$.

With the macrohomogeneity hypothesis and if the process of localization ensures uniqueness, one can define the concentration tensors in the form

$$
\begin{aligned}
\dot{\boldsymbol{\theta}} & =\mathcal{A}: \dot{\boldsymbol{\Theta}} \\
\operatorname{grad} v & =\mathcal{B}: G r a d V \\
\delta \otimes \delta & =\left\langle\mathcal{B}^{t}: \mathcal{A}\right\rangle .
\end{aligned}
$$

and the following general properties hold

$$
\begin{aligned}
\text { GradV } & =\left\langle\mathcal{A}^{t}: \operatorname{grad} v>\right. \\
\mathcal{A}: \mathcal{C} & =c^{o}: \mathcal{B} \\
\mathcal{C} & =\left\langle\mathcal{B}^{t}: c^{o}: \mathcal{B}\right\rangle .
\end{aligned}
$$

$\mathcal{A}$ and $\mathcal{B}$ must be independent of a rigid-body motion and possess some other properties due to objectivity. 


\section{THE STUDY OF THE POLYCRYSTAL}

\subsection{Introduction}

The deformation of a single crystal is determined by the energy embedded in the lattice and the plasticity due to slip governed by the Schmid rule or a viscoplastic potential associated with the normality rule.

If we refer to the triad of lattice vectors, the local deformation is described by additively splitting the velocity gradient into three parts (elastic rate gradient, rotation of the triad of lattice vector, plastic rate gradient), and the elastic constitutive law is determined by a linear relation between the elastic deformation rate and the objective Jaumann rate of the Cauchy stress in the lattice vectors rotation.

With the help of the Hill-Mandel macrohomogeneity hypothesis we can show that the macroscopic velocity gradient has the same decomposition as the microscopic one and that the stress-strain relation has the same form under an appropriate choice of the triad of vectors which give at each time the orientation of the polycrystal.

With this type of description, if the plastic part of the velocity gradient derives from a potential at the microscale, the macroscopic one derives from a viscoplastic potential, too.

After this description we analyse the symmetry or asymmetry of the rate boundaryvalue problem in terms of the velocity gradient and the rates of internal variables for different yielding surfaces.

For describing the behavior of polycrystals, we introduce macromechanical quantities, such as stresses, strains and elastic strains, which must be defined from the knowledge of the corresponding micromechanical quantities. To determine the constitutive law for polycrystals we use the Hill-Mandel method.

We consider the polycrystal as a macrohomogeneous body. In fact it contains several crystals with different shapes and orientations. Inside its volume the stress and strain are not uniform and are in equilibrium with homogeneous boundary conditions.

First we consider the behavior of an ideal single crystal. We study its evolution and give the decomposition into reversible and irreversible parts during the tranformation. The main idea is the fact that a single crystal is defined by a given triad of vectors, which describes the orientation of the crystal lattice, the reversible deformation being the deformation of the lattice. The plastic deformation due to glide on siip systems, perfectly defined relatively to this orientation, preserves the lattice structure and his frame.

The hypothesis of macrohomogeneity is formulated and the polycrystal behavior is analysed. The form of the macroscopic constitutive law is the same as the microscopic one and it is necessary to introduce a triad of vectors to ensure uniqueness of the decomposition of the rate. 


\subsection{The Single Crystal}

The constitutive law for a single crystal deforming by dislocation glide can be formulated according to the kinematic scheme based largely on the analysis of Mandel (1971) or Hill and Rice (1972), Zarka (1973) and Rice (1971).

The total deformation $\mathbf{F}$ can be decomposed as follows: a plastic deformation $\mathbf{F}_{p}$ given by a set of successively simple shears on active slip systems which are referred to a fixed lattice, followed by an elastic lattice deformation $\mathbf{F}_{e}$ which deforms and rotates the material and the lattice together so that

$$
\mathbf{F}=\mathbf{F}_{e} \cdot \mathbf{F}_{p}
$$

By unloading the single crystal and putting its lattice orientation back at its initial position one obtains a unique stress-free state $C_{K}$ which is defined by $\mathbf{F}_{p}$. It is obvious that the reversible part of the total deformation is determined by the Lagrangian strain tensor

$$
\boldsymbol{\Delta}_{K}=\frac{1}{2}\left(\mathbf{F}_{e}^{T} \cdot \mathbf{F}_{e}-\mathbf{I}\right) .
$$

Assuming that the hardening has no influence on the elastic properties, we can take on $C_{K}$ for the free energy density the form

$$
\psi\left(\boldsymbol{\Delta}_{k}, \gamma_{r}\right)=\frac{1}{2} \boldsymbol{\Delta}_{k}: \Lambda: \boldsymbol{\Delta}_{k}+h\left(\gamma_{r}\right),
$$

where $h$ is a function of the internal parameters $\gamma_{r}$, which are the ammounts of slip on each slip system (slip in the direction $m_{r}$ in the plane with normal $n_{r}$ )

The elastic constitutive law is given by :

$$
\frac{\boldsymbol{\pi}_{k}}{\rho_{k}}=\frac{\partial \psi}{\partial \boldsymbol{\Delta}_{k}}, \frac{A_{r}}{\rho_{k}}=-\frac{\partial \psi}{\partial \gamma_{r}}
$$

where $\pi_{k}$ is the Piola-Kirchhoff tensor referred to $C_{K}$ and $A_{r}$ the thermodynamic force associated with the internal parameter $\gamma_{r}$. Then the Cauchy stress $\mathbf{t}$

$$
\mathbf{t}=\rho \mathbf{F}_{e}^{T} \cdot \frac{\boldsymbol{\pi}_{k}}{\rho_{k}} \cdot \mathbf{F}_{e}
$$

is in equilibrium with the prescribed loading, and the dissipation rate is

$$
D=\frac{\mathbf{t}}{\rho}: \operatorname{grad} v-\dot{\psi}=\frac{\boldsymbol{\sigma}_{k}}{\rho_{k}}: \dot{\mathbf{F}}_{p} \cdot \mathbf{F}_{p}^{-1}+\frac{A_{r}}{\rho_{k}} \dot{\gamma}_{r} \geq 0 .
$$

As the plastic strain rate on $C_{K}$ is the sum of simple shear rates on the active slip systems, then

$$
\dot{\mathbf{F}}_{p} \cdot \mathbf{F}_{p}^{-1}=\sum_{r} \dot{\gamma}_{r} m_{r} \otimes n_{r}
$$


and the expression for the dissipation $D$ becomes

$$
D=\sum_{r}\left(n_{r} \cdot \frac{\boldsymbol{\sigma}_{k}}{\rho_{k}} \cdot m_{r}+\frac{A_{r}}{\rho_{r}}\right) \dot{\gamma}_{r} \geq 0
$$

with

$$
\frac{\boldsymbol{\sigma}_{k}}{\rho_{k}}=\mathbf{F}_{e}^{-1} \cdot \frac{\mathbf{t}}{\rho} \cdot \mathbf{F}_{e} .
$$

To determine the evolution of $\gamma_{r}$, one can define by

$$
f_{r}=n_{r} \cdot \frac{\sigma_{k}}{\rho_{k}} \cdot m_{r}+\frac{A_{r}}{\rho_{r}}-\tau_{o} \leq 0,
$$

the yielding function such that $\dot{\gamma}_{r} \geq 0$ if $f_{r}=0$, and $\dot{\gamma}_{r}=0$ in the other cases.

This is a generalized form of the Schmid law. With this definition, the elastic domain is a convex domain defined by a convex function of $\sigma_{k}$, and we have the law of normality

$$
\dot{\mathbf{F}}_{p} \cdot \mathbf{F}_{p}^{-1}=\sum_{r} \dot{\gamma}_{r} \frac{\partial f_{r}}{\partial \sigma_{k}} .
$$

The total plastic gradient rate is determined on $C_{K}$; its symmetric and antisymmetric parts are given by the plastic potentials $f_{r}$.

In the case of small elastic strains $\left(\mathbf{F}_{e}=\mathbf{S}\right.$.R with $\mathbf{S}=\mathbf{I}+\boldsymbol{\varepsilon}$ and $\left.\|\boldsymbol{\varepsilon}\| \ll 1\right)$ the local velocity gradient has the form

$$
g r a d v=\mathbf{d}_{e}+\boldsymbol{\omega}^{d}+\mathbf{R} \cdot \dot{\mathbf{F}}_{p} \cdot \mathbf{F}_{p}^{-1} \cdot \mathbf{R}^{-1},
$$

and the objective stress-strain rate relationship in the actual state is determined by the equation (Mandel, 1971; Halphen, 1975; Stolz, 1982)

$$
\frac{D}{D t} \frac{\mathbf{t}}{\rho}=\Lambda^{a}: \mathbf{d}_{\epsilon}
$$

where $D\left(\frac{\mathrm{t}}{\rho}\right) / D t$ is the Jaumann derivative of $\frac{\mathrm{t}}{\rho}$ in the rotation $\boldsymbol{\omega}^{d}$ of the lattice (Mandel, 1982). The instantaneneous elastic moduli are the convected ones from the stress free state by the clastic deformation $\mathbf{F}_{e}$.

$$
\Lambda_{i j k l}^{a}=\Lambda_{\alpha \beta \gamma \delta} \mathbf{F}_{i \alpha}^{e} \mathbf{F}_{j \beta}^{e} \mathbf{F}_{k \gamma}^{e} \mathbf{F}_{l \delta}^{e} .
$$

\subsection{The Macrohomogeneity Hypothesis}

We must first consider the equation of localization. From a formal point of view, the equation is obtained in thermodynamical form by replacing the strain by the deformation gradient, and the stress $\mathbf{t}$ by the nominal stress in the expression of the energy. 'The macrohomogeneity condition can be generalized as follows: for any gradient 
field $\mathbf{f}$, which is kinematically admissible with $\mathbf{F}=\langle\mathbf{f}>$ in the mode of localization and any nominal stress field $\theta$ statically admissible with $\Theta$ in the mode, we have $\langle\mathbf{f}: \boldsymbol{\theta}\rangle=\mathbf{F}: \boldsymbol{\Theta}$.

This equation can be expressed by choosing the present state as the reference configuration. The rate of the nominal stress can be expressed in terms of the Cauchy stress or in terms of the convective stress rate

$$
\begin{gathered}
\dot{\boldsymbol{\theta}}=\dot{\mathbf{t}}+\operatorname{gradv} \cdot \mathbf{t}+\mathbf{t} d i v v, \\
D_{c}\left(\frac{\mathbf{t}}{\rho}\right)=\stackrel{\circ}{(-)}+\operatorname{grad} v \cdot \frac{\mathbf{t}}{\rho}+\frac{\mathbf{t}}{\rho} \cdot \operatorname{grad}^{T} v, \\
\dot{\boldsymbol{\theta}}=\rho D_{c}\left(\frac{\mathbf{t}}{\rho}\right)+\mathbf{t} \cdot \operatorname{grad}^{T} v .
\end{gathered}
$$

Since the Cauchy stress $\mathbf{t}$ is statically admissible with $\mathbf{T}=\langle\mathbf{t}\rangle$ in the mode of localization, for any $v$ kinematically admissible with $V=\operatorname{GradV} . X, X \in \partial \Omega$, we have, noting also that $G r a d V=<\operatorname{grad} v>$

$$
<t^{i k} v_{j, k}>=\frac{1}{V} \int_{\partial \Omega} t_{i k} v_{j} n_{k} d a=T_{i k} V_{j, k} .
$$

In the same way it is easy to show that

$$
<\rho>D_{c}\left(\frac{\mathbf{T}}{\rho}\right)=<\rho D_{c}\left(\frac{\mathbf{t}}{\rho}\right)>.
$$

On the elastic behavior. For an elastic behavior with modulus $\Lambda_{o}$, we define the pseudomodulus $c_{o}$ by

$$
\dot{\boldsymbol{\theta}}=c_{\circ}: \operatorname{grad} v, \quad D_{c}\left(\frac{\mathbf{t}}{\rho}\right)=\Lambda_{\circ}: \mathbf{d} .
$$

Considering the localization process associated with the local elasticity tensor $c_{o}$, in the case of stability of the clastic path, we can define the stress localization tensor $\mathcal{A}$, or the strain localization tensor $\mathcal{B}$ by

$$
\begin{aligned}
\dot{\boldsymbol{\theta}} & =\mathcal{A}: \dot{\boldsymbol{\Theta}} \\
\operatorname{grad} v & =\mathcal{B}: \text { GradV }
\end{aligned}
$$

\subsection{The Polycrystal as a Macrohomogeneous Body}

In the description of the elastoplastic behavior of single crystals, the velocity gradient is split into three terms: the elastic strain rate $\mathbf{d}_{e}$, the spin $\boldsymbol{\omega}^{\boldsymbol{d}}$ of the crystal lattice and the plastic part $\mathbf{g}_{p}$, which can be decomposed into a strain rate and a rotation rate

$$
\operatorname{grad} v=\mathbf{d}_{e}+\boldsymbol{\omega}^{d}+\mathbf{g}_{p} .
$$


To this deformation rate, we associate the convective stress rate defined relative to the stress free state of each single crystal by

$$
\rho D_{c}\left(\frac{\mathbf{t}}{\rho}\right)=\Lambda_{o}:\left(\mathbf{d}-\mathbf{d}_{p}\right)-\mathbf{g}_{p} \cdot \frac{\mathbf{t}}{\rho}-\frac{\mathbf{t}}{\rho} \cdot \mathbf{g}_{p}^{t} .
$$

Then, the rate of nominal stresses can be expressed in terms of the pseudo-modulus $c_{o}$

$$
\dot{\boldsymbol{\theta}}=c_{o}:\left(\operatorname{grad} v-\mathbf{g}_{p}\right)-\mathbf{g}_{p} \cdot \mathbf{t} .
$$

At the macroscopic level, we assume that a stress-free state exists. Then, $\mathbf{F}$ may be written as $\mathbf{F}=\mathbf{F}_{e} \cdot \mathbf{F}_{p}$, and this decomposition is unique if we specify an orientation for the polycrystal by the introduction of a triad of directors. Then we have

$$
G r a d V=\dot{\mathbf{F}} \cdot \mathbf{F}^{-1}=\dot{\mathbf{F}}_{e} \cdot \mathbf{F}_{e}^{-1}+\mathbf{F}_{e} \cdot \dot{\mathbf{F}}_{p} \cdot \mathbf{F}_{p}^{-1} \cdot \mathbf{F}_{e}^{-1}=G r a d V_{E}+\mathbf{G}_{p} .
$$

The local elastic response $\operatorname{gradv}_{E}$ corresponds to the macroscopic velocity gradient $G r a d V_{E}$ such that

$$
\begin{aligned}
\operatorname{GradV}_{E} & =<\operatorname{gradv}_{E}> \\
\operatorname{gradv}_{E} & =\mathcal{B}: \operatorname{GradV}_{E} \\
\dot{\boldsymbol{\theta}}_{E} & =c_{o}: \nabla v_{E}
\end{aligned}
$$

and the corresponding macroscopic stress rate is

$$
\dot{\boldsymbol{\Theta}}_{E}=<\dot{\boldsymbol{\theta}}_{E}>\text {. }
$$

By defining the velocity fields $v_{r}$ and $v_{o}$ as kinematically admissible fields respectively with $\mathbf{G}^{p}$ and with 0 , the nominal stress rates

$$
\begin{aligned}
& \dot{\boldsymbol{\theta}}_{r}=c_{o}:\left(\operatorname{grad}_{r}-\mathbf{g}_{p}\right), \\
& \dot{\boldsymbol{\theta}}_{o}=c_{o}: \operatorname{grad} v_{o}-\mathbf{g}_{p} \cdot \mathbf{t},
\end{aligned}
$$

are chosen to be statically admissible fields respectively with 0 and $\mathbf{G}^{p}$ in the mode. With this decomposition the following properties are established

$$
\begin{aligned}
\operatorname{grad} v & =\operatorname{gradv}_{E}+\operatorname{gradv}_{r}+\operatorname{grad}_{o} \\
\dot{\boldsymbol{\theta}} & =\dot{\boldsymbol{\theta}}_{E}+\dot{\boldsymbol{\theta}}_{r}+\dot{\boldsymbol{\theta}}_{o} \\
\dot{\boldsymbol{\Theta}} & =\mathcal{C}: G r a d V_{E}-\mathbf{G}_{p} \cdot \mathbf{T}
\end{aligned}
$$

where the pseudomodulus is

$$
\mathcal{C}=\left\langle\mathcal{B}^{t}: c_{o}: \mathcal{B}>\right.
$$


These equations imply the following connections between the stress rate and the reversible part of the deformation

$$
<\rho>D_{c}\left(\frac{\mathbf{T}}{\rho}\right)=\Lambda:\left(\operatorname{Grad} V-\mathbf{G}_{p}\right)-\mathbf{G}_{p} \cdot \mathbf{T}-\mathbf{T} \cdot \mathbf{G}_{p}^{T},
$$

which corresponds to the relation

$$
\mathbf{t}=\left(\operatorname{det} \mathbf{F}_{e}\right) \mathbf{F}_{e} \cdot \pi_{k} \cdot \mathbf{F}_{e}^{t} .
$$

So the elastic part of the total velocity gradient is associated with the Piola-Kirchhoff stress defined on the stress-free state of the polycrystal. The following properties are obtained by the use of the macrohomogeneity condition

$$
\begin{aligned}
\operatorname{GradV} & =<\mathcal{A}^{t}: \operatorname{grad} v> \\
\operatorname{GradV}_{E} & =<\mathcal{A}^{t}: \operatorname{gradv}_{E}>, \\
\mathbf{G}^{p} & =<\mathcal{A}^{t}: \operatorname{gradv}_{r}>.
\end{aligned}
$$

For any macroscopic field $V$, we define the local field $v$ such that $g r a d v^{\prime}=\mathcal{B}$ : $G r a d V^{\prime}$ and the associated rate of nominal stress during purely elastic behavior

$$
\dot{\boldsymbol{\theta}}^{\prime}=c_{o}: \operatorname{grad} v^{\prime}=\mathcal{A}: \dot{\Theta}^{\prime} .
$$

For this particular choice we note that

$$
<\dot{\boldsymbol{\theta}}^{\prime}:\left(\operatorname{grad} v_{r}-\mathbf{g}_{p}\right)>=\dot{\boldsymbol{\Theta}}^{\prime}:<\mathcal{A}^{t}:\left(\operatorname{grad} v_{r}-\mathbf{g}_{p}\right)>=<\dot{\boldsymbol{\theta}}_{r}: \operatorname{gradv} v^{\prime}>=0,
$$

and we have, finally

$$
\mathbf{G}_{p}=<\mathcal{A}^{t}: \mathbf{g}_{p}>.
$$

This result leads to a natural definition of the rotation of the triad of directors for the polycrystal, using the decomposition of the macroscopic velocity gradient. More precisely, this triad has the rate of rotation $\Omega^{d}$ given by

$$
\boldsymbol{\Omega}^{d}=\left[<\mathcal{A}^{t}:\left(\operatorname{grad} v-\mathbf{g}_{p}\right)>\right]_{A} .
$$

This result generalizes the result previously obtained by Mandel [12] ; in general, the rotation of the orientation of the polycrystal is not the mean value of the local rotation. We have shown that the macroscopic behavior of a polycrystal and that of the single crystal can be described by analogous equations. In order to describe this macroscopic behavior it is necessary to specify an orientation of the stress free-state, this orientation is given by a triad of vectors the rotation of which is defined by a constitutive law. 


\section{EVOLUTION LAW AND THE RATE BOUNDARY-VALUE PROB- LEM}

The amount of gliding $\dot{\gamma}_{r}$ is positive if a loading condition is verified $f_{r}=0$ and $\dot{f}_{r}=0$; in other cases $\dot{\gamma}_{r}=0$. The interpretation of the yield criterion is object of many discussions and can be interpreted in many ways ${ }^{1}$ :

(a) The normal $n_{r}$ to the slipping plane is not a material vector; then, the evolution of the resolved shear is reduced to

$$
\dot{f}_{r}=m_{r} \cdot \dot{\boldsymbol{\sigma}}_{k} \cdot n_{r}=0 .
$$

(b) The normal $n_{r}$ is a material vector, and is at each time the normal of the physical slipping plane ; in this case

$$
\begin{gathered}
\dot{n}_{r}=\dot{\mathbf{F}}_{p} \cdot \mathbf{F}_{p}^{-1} \cdot n_{r}-n_{r} \cdot\left\{\dot{\mathbf{F}}_{p} \cdot \mathbf{F}_{p}^{-1}\right\}_{s} \cdot n_{r} n_{r} \\
\dot{f}_{r}=n_{r} \cdot\left(\frac{\dot{\boldsymbol{\sigma}}_{k}}{\rho_{k}}-\dot{\mathbf{F}}_{p} \cdot \mathbf{F}_{p}^{-1} \cdot \frac{\boldsymbol{\sigma}_{k}}{\rho_{k}}\right) \cdot m_{r}=0
\end{gathered}
$$

In this case the thermodynamical force is associated with $\mathbf{F}_{p}$ and defined on $C_{K}$. The proof is straightforward : the total Lagrangian strain related to the initial configuration $C_{o}$ is

$$
\Delta=\mathbf{F}_{p}^{T} \cdot \Delta_{k} \cdot \mathbf{F}_{p}+\frac{1}{2}\left(\mathbf{F}_{p}^{T} \cdot \mathbf{F}_{p}-\mathbf{I}\right) .
$$

The energy becomes a function of $\Delta, \mathbf{F}_{p}$ and $\gamma_{r}$, such that

$$
\psi_{o}\left(\boldsymbol{\Delta}, \mathbf{F}_{p}, \gamma_{r}\right)=\psi_{k}\left(\boldsymbol{\Delta}_{k}, \gamma_{r}\right)
$$

and we have

$$
\boldsymbol{\Sigma}_{o}=\rho_{o} \frac{\partial \psi_{o}}{\partial \mathbf{F}_{p}}
$$

For the sake of simplicity we do not consider the hardening case in what follows.

The total potential energy is given by $\psi_{o}$, so that we have

$$
E\left(u, \mathbf{F}_{p}, \gamma_{r}\right)=\int_{\Omega} \rho_{o} \psi_{o}\left(\Delta, \mathbf{F}_{p}, \gamma_{r}\right) d \omega-\int_{\partial \Omega_{T}} T^{d} . u d s,
$$

where $T^{d}$ are the external forces prescribed on $\partial \Omega_{T}$. The equations of equilibrium are

$$
\frac{\partial E}{\partial u} \cdot \delta u=0 .
$$

\footnotetext{
${ }^{1}$ To obtain different forms of $\dot{f}_{r}$ one can adopt suitable forms for $\tau_{o}$. The case (a) corresponds to a constant value and the case (b) to the variation of the normal $n$.
} 
They can be differentiated with respect to time to obtain the rate equilibrium equations. Denoting the stress by $\boldsymbol{\pi}$, function of $\boldsymbol{\Delta}$ and $\mathbf{F}_{p}$,

$$
\boldsymbol{\pi}=\rho_{o} \frac{\partial \psi_{o}}{\partial \boldsymbol{\Delta}}, \boldsymbol{\alpha}_{r}=m_{r} \otimes n_{r},
$$

Deriving the equilibrium equation we obtain

$$
\int_{\Omega} \dot{\boldsymbol{\pi}}:\left(\mathbf{F}^{T} . \delta \mathbf{F}\right)+\boldsymbol{\pi}:\left(\dot{\mathbf{F}}^{T} . \delta \mathbf{F}\right) d \omega-\int_{\partial \Omega_{T}} \dot{T}^{d} . \delta u d a=0
$$

The stress rate $\dot{\pi}$ verifies the local behavior

$$
\dot{\boldsymbol{\pi}}=\psi_{, \Delta \Delta}: \dot{\Delta}+\psi_{, \Delta \mathbf{F}_{p}}: \mathbf{F}_{p}^{T} \cdot\left(\sum_{r} \dot{\gamma}^{r} \boldsymbol{\alpha}_{r}\right)
$$

where $\dot{\gamma}^{r} \geq 0$ if $f_{r}=0$ and $\dot{f}_{r}=0$. Then, we have :

$$
\begin{aligned}
& (a) 0=\left(\boldsymbol{\alpha}_{r}^{T} \cdot \mathbf{F}_{p}\right): \psi_{, \Delta \mathbf{F}_{p}}: \dot{\Delta}+H_{r s} \dot{\gamma}_{s}+\boldsymbol{\alpha}_{r}^{T}:\left(\sum_{s} \dot{\gamma}_{s} \boldsymbol{\alpha}_{s} \cdot \frac{\sigma_{k}}{\ell}\right) \\
& (b) 0=\left(\boldsymbol{\alpha}_{r}^{T} \cdot \mathbf{F}_{p}\right): \psi_{, \Delta \mathbf{F}_{p}}: \dot{\Delta}+H_{r s} \dot{\gamma}_{s}
\end{aligned}
$$

where $H_{r s}=\left(\boldsymbol{\alpha}_{r}^{T} \cdot \mathbf{F}_{p}\right): \psi_{, \mathbf{F}_{p} \mathbf{F}_{p}}:\left(\mathbf{F}_{p}^{T} \cdot \boldsymbol{\alpha}_{s}\right)$ is symmetric en $(\mathbf{r}, \mathbf{s})$.

It is clear that the case $(b)$ is a symmetric problem and that the case (a) is symmetric only for a simple plastic potential, as in the analysis due to Halphen (1975). Case (b) corresponds to the rate boundary-value problem in the theory of the plasticity of standard generalized materials.

Case of a polycrystal. The same equations are true for a polycrystal. The decomposition of the deformation gradient has the similar form

$$
g r a d v=\dot{\mathbf{F}}_{e} \cdot \mathbf{F}_{e}^{-1}+\mathbf{F}_{e} \cdot \dot{\mathbf{F}}_{p} \cdot \mathbf{F}_{p}^{-1} \cdot \mathbf{F}_{e}^{-1} .
$$

As previously, the reversibility is defined on the stress-free state and is characterized by the strain

$$
\boldsymbol{\Delta}_{k}=\frac{1}{2}\left(\mathbf{F}_{e}^{T} \cdot \mathbf{F}_{e}-\mathbf{I}\right) .
$$

The choice of the free energy $\psi$ is defined as in the case of the single crystal as function of the strain $\Delta_{k}$ and of the set of internal parameters $\alpha$ which describe in a global manner the internal state of the crystal, we assumed for the sake of simplicity that the hardening has no influence on the elasticity i.e. $\psi=\psi_{k}\left(\Delta_{k}\right)+\psi_{b}(\alpha)$. The dissipation has the form

$$
D=\frac{\mathbf{t}}{\rho}: \operatorname{grad} v-\dot{\psi}=\frac{\boldsymbol{\Sigma}_{k}}{\rho_{k}}: \dot{\mathbf{F}}_{p} \cdot \mathbf{F}_{p}^{-1}+A \cdot \dot{\alpha} \geq 0
$$


where $A=-\partial \psi / \partial \alpha$ and $\boldsymbol{\Sigma}_{k}$ has the same definition as above. We adopt the normality rule to define the irreversibility. Then

$$
\begin{aligned}
\dot{\mathbf{F}}_{p} \cdot \mathbf{F}_{p}^{-1} & =\mu \frac{\partial f}{\partial \frac{\boldsymbol{\Sigma}_{k}}{\rho_{k}}}=\mu \mathbf{N} \\
\dot{\alpha} & =\mu \frac{\partial f}{\partial A}=\mu a
\end{aligned}
$$

where $\mu \geq 0$ if $f\left(\frac{\Sigma_{k}}{\rho_{k}}, A\right)=0$. The consistency condition must be taken into account:

- if $f\left(\frac{\boldsymbol{\Sigma}_{k}}{\rho_{k}}, A\right)=0$ and $\dot{f}=0$, then $\mu \geq 0$,

- if $f\left(\frac{\boldsymbol{\Sigma}_{k}}{\rho_{k}}, A\right)=0$ and $\dot{f}<0$, then $\mu=0$.

This law of evolution could be rewritten as the inequation

$$
\dot{f}\left(\mu-\mu^{\prime}\right) \geq 0,
$$

defined on $K=\left\{\mu^{\prime} \mid \mu^{\prime} \geq 0\right.$ if $f\left(\frac{\boldsymbol{\Sigma}_{k}}{\rho_{k}}, A\right)=0$ and $\mu^{\prime}=0$ otherwise $\}$.

We can write the boundary-value problem on the initial configuration, through the definition of the potential energy:

Denoting by $\Psi\left(\Delta, \mathbf{F}_{p}, \alpha\right)$ the local free energy density $\psi\left(\boldsymbol{\Delta}_{k}, \alpha, t\right)$, the total potential energy is given by

$$
E\left(u, \mathbf{F}_{p}, \alpha\right)=\int_{\Omega} \rho_{o} \Psi\left(\Delta, \mathbf{F}_{p}, \alpha\right) d \omega-\int_{\partial \Omega_{T}} T^{d} \cdot u d s,
$$

where $T^{d}$ are the external forces prescribed on $\partial \Omega_{T}$. The equations of equilibrium are

$$
\frac{\partial E}{\partial u} . \delta u=0 .
$$

They can be differentiated with respect to time to obtain the rate equilibrium equations. Denoting the stress by $\pi$, function of $\Delta$ and $\mathbf{F}_{p}$,

$$
\boldsymbol{\pi}=\rho_{o} \frac{\partial \Psi}{\partial \Delta}, A=-\rho_{o} \frac{\partial \Psi}{\partial \alpha}, \boldsymbol{\Sigma}=-\rho_{o} \frac{\partial \Psi}{\partial \mathbf{F}_{p}},
$$

and time differentiating the equilibrium equation, we obtain

$$
\int_{\Omega} \dot{\boldsymbol{\pi}}:\left(\mathbf{F}^{T} . \delta \mathbf{F}\right)+\pi:\left(\dot{\mathbf{F}}^{T} . \delta \mathbf{F}\right) d \omega-\dot{\lambda} \int_{\partial \Omega_{T}} T^{d} . \delta u d a=0
$$

The stress $\dot{\pi}$ verifies the local behavior

$$
\dot{\pi}=\Psi_{, \Delta \Delta}: \dot{\Delta}+\Psi_{, \Delta \mathbf{F}_{p}}: \dot{\mathbf{F}}_{p}
$$


where $\dot{\mathbf{F}}_{p}$ is defined by the consistency condition

$$
\begin{aligned}
0 & \leq\left(\mu-\mu^{\prime}\right)\left[\left(\mathbf{N} . \mathbf{F}_{p}\right)^{T}: \Psi_{, \mathbf{F}_{p} \Delta}: \dot{\Delta}+\left(\mathbf{N} . \mathbf{F}_{p}\right)^{T}: \Psi_{\left.{ }_{\mathbf{F}_{p} \mathbf{F}_{p}}:\left(\mathbf{N} . \mathbf{F}_{p}\right) \mu+\mathbf{H} \mu\right]}\right. \\
\mathbf{H} & =a^{T} \cdot \Psi_{, \alpha \alpha} \cdot a
\end{aligned}
$$

Taking the average of the inequation on the whole volume, the boundary-value problem assumes the global form

$$
\begin{gathered}
\int_{\Omega}\left(\mathbf{F}^{T} . \delta \mathbf{F}\right):\left(\Psi_{, \Delta \Delta}: \dot{\Delta}+\Psi_{, \Delta \mathbf{F}_{p}}:\left(\mathbf{N F}_{p}\right) \mu\right)+\pi:\left(\dot{\mathbf{F}}^{T} . \delta \mathbf{F}\right) d \omega-\int_{\partial \Omega_{T}} \dot{T}^{d} . \delta u d a=0, \\
\int_{\Omega}\left(\mu-\mu^{\prime}\right)\left[\left(\mathbf{N} . \mathbf{F}_{p}\right)^{T}: \Psi_{\mathbb{F}_{p} \Delta}: \dot{\Delta}+\left(\mathbf{N} . \mathbf{F}_{p}\right)^{T}: \Psi_{, \mathbf{F}_{p} \mathbf{F}_{p}}:\left(\mathbf{N} . \mathbf{F}_{p}\right) \mu+\mathbf{H} \mu\right] d \omega \geq 0 .
\end{gathered}
$$

By introducing the global potential

$$
\begin{aligned}
J(v, \mu)= & \int_{\Omega} \frac{1}{2}\left(\dot{\Delta}: \Psi_{, \Delta \Delta}: \dot{\Delta}+\dot{\Delta}: \Psi_{, \Delta \mathbf{F}_{p}}:\left(\mathbf{N F}_{p}\right) \mu\right) d \omega \\
+\int_{\Omega} \frac{1}{2} \mu\left[\left(\mathbf{N} . \mathbf{F}_{p}\right)^{T}:\right. & \left.\Psi_{, \mathbf{F}_{p} \Delta}: \dot{\Delta}+\left(\mathbf{N} \cdot \mathbf{F}_{p}\right)^{T}: \Psi_{\mathbf{F}_{p} \mathbf{F}_{p}}:\left(\mathbf{N}_{\mathbf{F}} \mathbf{F}_{p}\right) \mu+\mathbf{H} \mu\right] d \omega \\
& +\int_{\Omega} \frac{1}{2} \dot{\mathbf{F}}^{T} \cdot \pi \cdot \dot{\mathbf{F}} d \omega-\dot{\lambda} \int_{\partial \Omega_{T}} T^{d} . v d a
\end{aligned}
$$

Then, the solution $(v, \mu)$ verifies the variational inequality

$$
\frac{\partial J}{\partial v} \bullet\left(v^{\prime}-v\right)+\frac{\partial J}{\partial \mu} \bullet\left(\mu^{\prime}-\mu\right) \geq 0,
$$

on the set of admissible fields $\left(v^{\prime}, \mu^{\prime}\right)$ such that

$v^{\prime}$ is K.A. with the displacement boundary conditions and $\mu^{\prime}$ is positive only where the yielding function is reached. Let the plastic zone be determined at the actual position

$$
\Omega_{p}=\left\{x \in \Omega / f\left(\frac{\boldsymbol{\Sigma}_{k}}{\rho_{k}}, A\right)=0\right\},
$$

then the admissible fields are defined on the set

$$
\begin{aligned}
& \mathcal{K}=\left\{\left(v^{\prime}, \mu\right) / v^{\prime}=v^{d} \text { over } \partial \Omega_{u},\right. \\
& \left.\mu^{\prime} \geq 0 \text { over } \Omega_{p}, \mu^{\prime}=0 \text { otherwise }\right\}
\end{aligned}
$$

On the actual state, the rate boundary-value problem is symmetric and the fields verify the variational inequality

$$
\frac{\partial J}{\partial v} \bullet\left(v^{\prime}-v\right)+\frac{\partial J}{\partial \mu} \bullet\left(\mu^{\prime}-\mu\right) \geq 0,
$$


where $J$ is defined as

$$
\begin{aligned}
J= & \frac{1}{2} \int_{\Omega}\left[(\mathbf{D}-\mu \mathbf{n}): C:(\mathbf{D}-\mu \mathbf{n})+\operatorname{grad} v \cdot \mathbf{t} \cdot \operatorname{grad}^{t} v\right] d \omega \\
& +\int_{\Omega} \frac{1}{2} \mu\left(\mathbf{H}^{\prime}+2\left(\frac{\mathbf{t}}{\rho} \cdot \mathbf{n}\right) \cdot \mathbf{n}\right) \mu d \omega-\int_{\partial \Omega} \dot{T}^{d} . v d a .
\end{aligned}
$$

where $\mathbf{H}^{\prime}=\mathbf{n}: C: \mathbf{n}+\mathbf{H}$, and $\mathbf{n}=\mathbf{F}_{e} \cdot \mathbf{N} \cdot \mathbf{F}_{e}^{-1}$.

The moduli of elasticity are convected from the stress-free state and the above formula shows that the behavior is hypoelastic in the sense of Hill.

Many other formulations can be derived in the case of isotropic elasticity, and classical formulations are then recovered.

\section{References}

[1] Bui, H. D.: Etude de l'évolution de la frontière du domaine élastique avec l'écrouissage et relation de comportement élastoplastique des métaux cubiques, Thèse de Doctorat, Paris VI, 1969.

[2] Francfort, G., Nguyen, Q. S. et Suquet, P.: Thermodynamique et lois de comportement thermomécanique, Comptes Rendus Acad. Sci. de Paris, II, 296 (1983), $1007-10$.

[3] Germain, P., Nguyen, Q. S. and Suquet, P.: Continuum thermodynamics, J. Appl. Mech., 50 (1983), 1010-1020.

[4] Halphen, B.: Sur le champ des vitesses en thermoplasticité finie, Int. J. Solids Struct., 11 (1975), 947-60.

[5] Hashin, Z.: Analysis of composite materials, J. Appl. Mech., 50 (1983), 481- 485.

[6] Hill, R.: Generalised constitutive relations for incremental deformation of metal crystals by multislip, J. Mech. Phys. Solids, 14 (1966), 95-102.

[7] Hill, R.: The essential structure of constitutive laws of metal composites and polycristals, J. Mech. Phys. Solids, 15 (1967), 79-95.

[8] Hill, R. and Rice, J.: Constitutive analysis of elastic-plastic crystals at arbitrary strain, J. Mech. Phys. Solids, 20 (1972), 401-413.

[9] Kröner, E.: Linear properties of random media, Proc. 15th Colloq. Groupe Français de Rhéologie, 1980. 
[10] Mandel, J.: Contribution théorique à l'étude de l'écrouissage et des lois de l'écoulement plastique, Proc. 11th Int. Congr. Appl. Mech., Springer-Verlag, Berlin, 1964.

[11] Mandel, J.: Plasticité Classique et Viscoplasticité, CISM, Udine, Springer-Verlag, 1971.

[12] Mandel, J.: Sur la définition d'un repère privilegié pour l'étude des transformations anélastiques d'un polycristal, J. Mécanique Théorique et Appliquée, 1 (1982), 7-23.

[13] Nguyen, Q. S.: Bifurcation et stabilité des systèmes irréversibles obéissant au principe de dissipation maximale, J. Mécanique Théorique et Appliquée, 3 (1984), No. 7, 41-61.

[14] Rice, J.: Inelastic constitutive relations for solids: an internal variable theory and its application to crystal plasticity, J. Mech. Phys. Solids, 19 (1971), 433-455.

[15] Sanchez Palencia, E.: Comportement local et macroscopique d'un type de matériaux plastiques hétérogènes, Int. J. ENGNG. SCi., 12 (1974), 331-351.

[16] Ștolz, C.: Contribution à l'étude des grandes transformations en élastoplasticité, Doctorat ENPC, Paris, 1982.

[17] Stolz, C.: Etude des milieux à configuration physique et applications, Comptes Rendus Acad. Sci. de Paris, II, 299 (1984), 1153-1155.

[18] Stolz, C.: On relationships between micro- and macro-scales for particular cases of non linear behavior in heterogeneous media, in Yielding Damage and Failure of Anisotropic Solids, EGF5 (Ed. J.P. Boehler), 1989, Mechanical Engineering Publication, London.

[19] Stolz, C.: Study of the constitutive law for a polycristal and analysis of rate boundary value problem in finite elastoplasticity, in Large Deformations of Solids, Ed. Zarka, Gittus and Nemat Nasser, Elsevier Applied Science, 1985.

[20] Stolz, C.: General relationships between micro and macro scales for the non linear behavior of heterogneous media, in Modelling Small Deformations of Polycrystals, Ed J. Zarka and J. Gittus, Elsevier Science Publishers, 89-115, 1983.

[21] Willis, J. 1.: Overall Properties of Composites, in Advances in Applied Mechanics, Vol. 21, C. S. Yih (Ed.), Academic Press, New York, 1981.

[22] Zarka, J. : Etude du comportement des monocristaux métalliques, J. Mécanique Théorique et Appliquée, 12, (1973). 